\title{
Inflatonic solitons in running mass inflation
}

\author{
Kari Enqvist, ${ }^{1,2}$ Shinta Kasuya, ${ }^{2}$ and Anupam Mazumdar ${ }^{3}$ \\ ${ }^{1}$ Department of Physical Sciences, P.O. Box 64, FIN-00014, University of Helsinki, Finland \\ ${ }^{2}$ Helsinki Institute of Physics, P.O. Box 64, FIN-00014, University of Helsinki, Finland \\ ${ }^{3}$ The Abdus Salam International Centre for Theoretical Physics, I-34100, Trieste, Italy
}

(Received 27 June 2002; published 9 August 2002)

\begin{abstract}
The inflaton condensate associated with a global symmetry can fragment into quasistable $Q$ balls, provided the inflaton oscillations give rise to an effective equation of state with negative pressure. We study chaotic inflation with a running inflaton mass and show that, depending on the sign of the radiative mass correction, the process of fragmentation into inflatonic $Q$ balls can actually take place even though no net charge exists. If the main decay channel of the $Q$ ball is to fermions, the universe will be reheated slowly via surface evaporation.

DOI: 10.1103/PhysRevD.66.043505

PACS number(s): 98.80.Cq, 11.27.+d, 11.30.Fs
\end{abstract}

\section{INTRODUCTION}

Reheating of the universe, in which the inflaton decay product provide a thermal bath of radiation, is one of the cornerstones of any inflationary model. At the end of inflation the coherent inflaton condensate may decay either perturbatively [1], one quantum at the time, or nonperturbatively [2] via a parametric resonance in a collective process denoted as preheating. Usually in the literature reheating has been considered to be a volume effect so that it tends to be relatively fast, and as a consequence, the reheat temperature is relatively high. In supersymmetric models this has turned out to be somewhat problematic because a large reheat temperature generates a large gravitino abundance which is in conflict with the successful big bang nucleosynthesis [3].

However, recently we have pointed out [4] a novel possibility in which reheating could be realized as a surface effect, noting that the evaporation rate from a surface is always suppressed by the area of the evaporating surface. Therefore the larger the surface, the smaller the reheat temperature of the universe. Such a situation can be achieved if the zeromode inflaton condensate does not remain spatially coherent but breaks down into quasistable lumps. This can happen if the inflaton field carries a global $U(1)$ charge which protects the lumps; for instance, the inflaton could be a complex rather than a real scalar field. The ground state of such a condensate lump would be a nontopological soliton called $Q$ ball [5], the properties of which depend on the exact form of the scalar potential. A general requirement for its existence is that the scalar potential should grow slower than $|\Phi|^{2}$.

Inflaton condensate fragmentation has recently been discussed in the context of hybrid inflation models both numerically [6] and analytically [7]. However, in these cases the shape of the potential does not render the condensate lumps quasistable and their appearance is merely a part of the complicated preheating dynamics. In fact, there they appear to make reheating even more effective rather than slowing it down [7].

$Q$ ball formation has been considered in detail in the context of the MSSM flat direction condensates (Affleck-Dine condensates [8]), and can be either completely stable [9-13] if SUSY is broken by gauge mediation, or quasi-stable
[14-16] if SUSY is broken by gravity mediation. In the Affleck-Dine case the condensate is charged up by a helical motion in the field space, and fragmentation takes place when the fluctuations in the real and the imaginary directions grow to become of order one.

In this paper we consider the simple case of the chaotic inflation but with a complex inflaton field (which is rather more natural) and a running inflaton mass, such as might be generated radiatively by the Yukawa and/or gauge couplings of the inflaton to other fields. We demonstrate numerically that at the end of inflation the inflaton condensate fragments into lumps and forms $Q$ balls. The initial fragmentation occurs because of the negative pressure the condensate feels during the inflaton oscillations. We show that the fluctuations in the imaginary direction grow because of the mode-mode coupling between the real and the imaginary parts and thus provide the motion in field space necessary for solitogenesis.

The paper is organized as follows. In Sec. II we describe $Q$ balls in a running mass potential and discuss how the fluctuations grow for a certain running mass inflaton potential in Sec. III. In Sec. IV we study $Q$-ball formation due to mode-mode couplings by lattice simulations. Section V is devoted to the implications of $Q$-ball formation and a discussion on the reheat temperature. In Sec. VI we conclude with a brief discussion highlighting the results and describing future prospects.

\section{II. $Q$ BALLS IN THE RUNNING MASS INFLATON POTENTIAL}

If the inflaton has couplings to other fields, then, in general, the inflaton mass should receive radiative corrections [17]. The simplest chaotic inflation potential with a running inflaton mass could then be written as

$$
V=m^{2}|\Phi|^{2}\left[1+K \log \left(\frac{|\Phi|^{2}}{M^{2}}\right)\right],
$$

where the coefficient $K$ could be negative or positive, and $m$ is the bare mass of the inflaton. The logarithmic correction to 
the mass of the inflaton is something one would expect to arise because of the possible Yukawa and/or gauge couplings to other fields.

Since we assume that $|K| \ll 1$, the second term in the bracket does not affect the inflaton dynamics or generation of perturbations much so that with a running mass the universe would experience the same chaotic inflationary stage as with the usual $m^{2}|\Phi|^{2}$ potential. However, as we shall see, the values $K<0$ lead to interesting cosmological consequences. Though it is not pertinent, we note that the potential Eq. (1) can be generated in a supersymmetric theory if the inflaton has a gauge coupling $[15,4]$. This is due to the fact that the gaugino loops contribute as a negative correction which dominates. In this case the value of $K$ is given by

$$
K \sim-\frac{\alpha}{8 \pi} \frac{m_{1 / 2}^{2}}{m_{\tilde{\ell}}^{2}}
$$

where $m_{1 / 2}$ is the gaugino mass and $m_{\tilde{\ell}}$ denotes the mass of slepton. It is also possible to obtain the potential Eq. (1) in a nonsupersymmetric (or in broken SUSY) theory, provided the fermions live in a larger representation than the bosons. In this latter situation the value of $K$ is determined by the Yukawa coupling [4]

$$
K=-C \frac{h^{2}}{16 \pi^{2}} .
$$

Here $C$ is the total number of fermionic loops, and $h$ is the Yukawa coupling. As long as $|K| \ll 1$, the dominant contribution to the potential comes from $m^{2}|\Phi|^{2}$ term during inflation, and inflationary slow roll conditions are satisfied as in the case of the standard chaotic model. The generation of the amplitude of the density perturbations are constrained by the COBE normalization, which implies

$$
\frac{\delta \rho}{\rho} \sim \frac{m}{M_{\mathrm{P}}} \sim 10^{-5}
$$

which results in $m \sim 10^{13} \mathrm{GeV}$.

The most important virtue of the inflationary potential Eq. (1) is that it respects a global $U(1)$ symmetry, and for a negative $K$, the potential is shallower than $m^{2}|\Phi|^{2}$. It has been well known that such a potential admits a $Q$-ball solution [15]. This means that the energy of a $Q$-ball configuration is less than a collection of free scalars carrying an equivalent charge. The field configuration is given by $\Phi(x, t)=\left(e^{i \omega t} \phi(x)\right) / \sqrt{2}$ while its energy and charge read

$$
\begin{aligned}
& E=\int d^{3} x\left[\frac{1}{2} \omega^{2} \phi^{2}+\frac{1}{2}(\nabla \phi)^{2}+V(\phi)\right], \\
& Q=\omega \int \phi^{2} d^{3} x .
\end{aligned}
$$

Here we assume that the potential $V(\phi)$ has a global mini- mum at the origin and is invariant under a global $U(1)$ transformation.

Depending on the slope of the potential it is possible to have an energetically favorable state in the form of $Q$ balls. The energy of the $Q$ ball is $E=\mu Q$, where $\mu$ lies in the range $\mu_{0} \leqslant \mu<m_{0}$, and $\mu_{0}$ and $m_{0}$ are given by

$$
\mu_{0}=\min \left(\sqrt{\frac{2 V(\phi)}{\phi^{2}}}\right), m_{0}=\sqrt{V^{\prime \prime}(0)} .
$$

The $Q$ ball is stable against decay into other particles if $\mu q$ $<m_{\text {decay }}$, where $m_{\text {decay }}$ is the smallest mass of the particles which shares the same $U(1)$ charge as the complex field $\Phi$, and $q$ is the charge carried by the particle. In this case, a $Q$ ball exists forever. On the other hand, if the stability condition does not hold, $Q$ balls will eventually decay into lighter particles. However, if the (final) decay products are pairs of fermions, the decay should proceed by the evaporation of charges through the surface of $Q$ balls because of the Pauli blocking [18]. Thus, inflatonic $Q$ balls with a large enough charge could live very long, in which case reheating may take place very slowly, as we shall discuss.

The potential of the form Eq. (1) generically forms a thick-wall $Q$ ball with a Gaussian profile [15]

$$
\phi(r) \propto \exp \left(-|K| m^{2} r^{2}\right)
$$

so that the size of such a $Q$ ball can be estimated by

$$
R \simeq|K|^{-1 / 2} m^{-1}
$$

The energy of a $Q$ ball can be approximately given by

$$
E \simeq m Q
$$

and the total charge accumulated by a $Q$ ball is given by

$$
Q \simeq \omega \phi_{0}^{2} V \sim \frac{4 \pi}{3} m \phi_{0}^{2} R^{3}
$$

where $\phi_{0}$ is the field value at which the $Q$ ball forms, and $V \sim 4 \pi R^{3} / 3$ is the volume of the $Q$ ball.

\section{AMPLIFICATION OF FLUCTUATIONS}

In the context of Affleck-Dine baryogenesis, the homogeneous mode is rotating around the origin in the potential. In such a case fluctuations tend to grow when on the average there is a negative pressure, which can be achieved in the potential Eq. (1) when $K<0$ [19,20]. Expanding the potential in Eq. (1) one finds

$$
V(\phi) \simeq \frac{1}{2} m^{2} \phi^{2}\left(\frac{\phi^{2}}{2 M^{2}}\right)^{K} \propto \phi^{2+2 K}
$$


where we assume $|K| \ll 1$, since this term is actually a correction to a tree level potential. The equation of state for a field rotating in such a potential is then given by ${ }^{1}$

$$
p \simeq \frac{K}{2+K} \rho \simeq-\frac{|K|}{2} \rho,
$$

where $p$ and $\rho$ is a pressure and energy density of the scalar field, respectively. Therefore, negative value of $K$ is crucial for negative pressure, which is the core of our discussion. Notice the difference between this case and the usual chaotic potential $V=m^{2}|\Phi|^{2}$, where on the average the oscillating inflaton condensate acts as a pressureless fluid.

When the homogeneous mode is rotating in the potential, it is appropriate to write a complex field as $\Phi=\left(\phi e^{i \theta}\right) / \sqrt{2}$, and decompose it into a homogeneous part and fluctuations: $\phi \rightarrow \phi+\delta \phi$ and $\theta \rightarrow \theta+\delta \theta$. The equations of motion of the field read as $[10,15]$

$$
\begin{array}{r}
\ddot{\phi}+3 H \dot{\phi}-\dot{\theta}^{2} \phi+V^{\prime}(\phi)=0, \\
\phi \ddot{\theta}+3 H \phi \dot{\theta}+2 \dot{\phi} \dot{\theta}=0,
\end{array}
$$

for the homogeneous mode, and

$$
\delta \ddot{\phi}+3 H \delta \dot{\phi}-2 \dot{\theta} \phi \delta \dot{\theta}-\dot{\theta}^{2} \delta \phi-\frac{\nabla^{2}}{a^{2}} \delta \phi+V^{\prime \prime}(\phi) \delta \phi=0,
$$

$$
\phi \delta \ddot{\theta}+3 H \phi \delta \dot{\theta}+2(\dot{\phi} \delta \dot{\theta}+\dot{\theta} \delta \dot{\phi})-2 \frac{\dot{\phi}}{\phi} \dot{\theta} \delta \phi-\phi \frac{\nabla^{2}}{a^{2}} \delta \theta=0,
$$

$$
\begin{gathered}
\frac{3 K}{2+K} H \dot{\alpha}+\ddot{\alpha}+\dot{\alpha}^{2}+\frac{k^{2}}{a^{2}}+2 m^{2} K a^{-6 K /(2+K)} \\
\frac{2 m \dot{\alpha}}{\phi_{0}}
\end{gathered}
$$

where we set $a_{0}=1$.

Let us neglect the cosmological expansion and assume $\ddot{\alpha} \ll \dot{\alpha}^{2}$ for simplicity. Then, Eq. (23) will be simplified as

$$
\left|\begin{array}{cc}
\dot{\alpha}^{2}+\frac{k^{2}}{a^{2}}+2 m^{2} K & -2 m \phi_{0} \dot{\alpha} \\
\frac{2 m \dot{\alpha}}{\phi_{0}} & \dot{\alpha}^{2}+\frac{k^{2}}{a^{2}}
\end{array}\right|=0 .
$$

\footnotetext{
${ }^{1}$ Because in the Affleck-Dine case the exact motion of the field is helical and hence strictly speaking nonperiodic, the equation of state actually oscillates [21].
}

for the fluctuations, and

$$
\begin{aligned}
& V^{\prime}(\phi)=m^{2} \phi\left[1+K+K \log \left(\frac{\phi^{2}}{2 M^{2}}\right)\right], \\
& V^{\prime \prime}(\phi)=m^{2}\left[1+3 K+K \log \left(\frac{\phi^{2}}{2 M^{2}}\right)\right] .
\end{aligned}
$$

Equation (14) represents the conservation of the charge (or number) within the physical volume: $\dot{\theta} \phi^{2} a^{3}=$ const. Since the energy density of the scalar field dominates the universe, the homogeneous part of the field evolves as

$$
\begin{gathered}
\phi(t) \simeq\left(\frac{a(t)}{a_{0}}\right)^{-3 /(2+K)} \phi_{0}, \\
\dot{\theta}^{2}(t) \simeq\left(\frac{a(t)}{a_{0}}\right)^{-6 K /(2+K)} m^{2} .
\end{gathered}
$$

We are now going to find the most amplified mode. To this end, we take the solutions in the form

$$
\begin{gathered}
\delta \phi=\left(\frac{a(t)}{a_{0}}\right)^{-3 /(2+K)} \delta \phi_{0} e^{\alpha(t)+i k x}, \\
\delta \theta=\left(\frac{a(t)}{a_{0}}\right)^{-3 K /(2+K)} \delta \theta_{0} e^{\alpha(t)+i k x} .
\end{gathered}
$$

If $\dot{\alpha}$ is real and positive, these fluctuations grow exponentially, and go nonlinear to form $Q$ balls. Putting these forms into Eqs. (15) and (16), we get the following condition for nontrivial $\delta \phi_{0}$ and $\delta \theta_{0}$,

$$
\begin{gathered}
-2 m a^{-6 K /(2+K)} \phi_{0}\left(-\frac{3 K}{2+K} H+\dot{\alpha}\right) \\
+\dot{\alpha}^{2}+\frac{k^{2}}{a^{2}}+\frac{3 K}{2+K}\left[(4-3 K) H^{2}-\frac{\ddot{a}}{a}-H \dot{\alpha}\right]
\end{gathered} \mid=0,
$$

In order for $\dot{\alpha}$ to be real and positive, we must have

$$
\frac{k^{2}}{a^{2}}\left(\frac{k^{2}}{a^{2}}+2 m^{2} K\right)<0 .
$$

As we are considering negative $K$, an instability band exists. This is because the rotating inflaton field in the potential flatter than $\phi^{2}$ has a negative pressure. Thus the instability band should be found in the range

$$
0<\frac{k^{2}}{a^{2}}<\frac{k_{\max }^{2}}{a^{2}} \equiv 2 m^{2}|K| .
$$




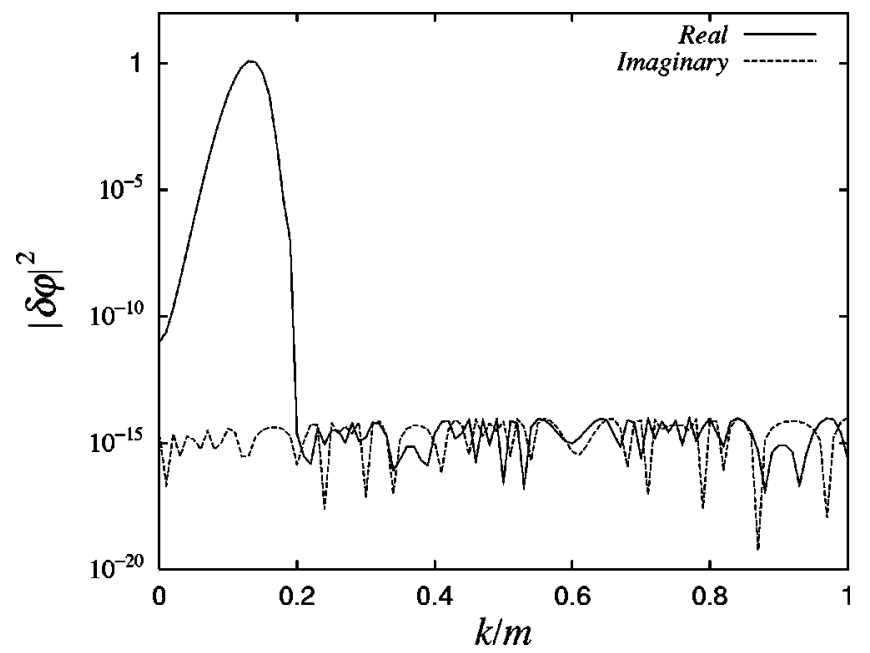

FIG. 1. Instability bands when the homogeneous mode is just oscillating along the real direction in the potential (1). Solid and dashed lines denote the fluctuations in the real and imaginary directions, respectively. Here we take $K=-0.02$.

We can easily derive that the most amplified mode lies at about the center of the band: $\left(k_{\text {most }} / a\right)^{2} \simeq m^{2}|K|(1-|K| / 4)$, and the maximum growth rate is $\dot{\alpha}_{\text {most }}=|K| m_{3 / 2} / 2{ }^{2}$

After inflation the inflaton field is just oscillating around the origin of its potential. It is thus more appropriate to decompose the inflaton field into its real and imaginary parts for analyzing the dynamics when the motion is non-circular. For numerical calculations it is convenient to take all the variables to be dimensionless, so we normalize as $\varphi$ $=\phi / m, \quad \tilde{k}=k / m, \tau=m t$, and $\xi=m x$. Writing $\varphi=\left(\varphi_{1}\right.$ $\left.+i \varphi_{2}\right) / \sqrt{2}$, we get the equations for the homogeneous mode as

$$
\varphi_{i}^{\prime \prime}+3 h \varphi_{i}^{\prime}+\left[1+K+K \log \left(\frac{\varphi_{1}^{2}+\varphi_{2}^{2}}{2 M^{2}}\right)\right] \varphi_{i}=0,
$$

where $h=H / m, i=1,2$, and the prime denotes the derivative with respect to $\tau$, and, for the fluctuations,

$$
\left[\frac{d^{2}}{d \tau^{2}}+3 h \frac{d}{d \tau}+\frac{\tilde{k}^{2}}{a^{2}}+V_{i j}\right]\left(\begin{array}{c}
\delta \varphi_{1} \\
\delta \varphi_{2}
\end{array}\right)=0
$$

where $V_{i j}$ denotes the second derivative with respect to $\varphi_{i}$ and $\varphi_{j}$, and explicitly written as

$$
V_{i i}=1+K+K \log \left(\frac{\varphi_{1}^{2}+\varphi_{2}^{2}}{2 M^{2}}\right)+2 K \frac{\varphi_{i}^{2}}{\varphi_{1}^{2}+\varphi_{2}^{2}},
$$

\footnotetext{
${ }^{2}$ The coefficients of these results are slightly different from the previous estimations $[16,13]$ because these were based on the approximation $\dot{\theta}^{2} \approx m^{2}$. Replacing this with the more accurate $\dot{\theta}^{2}$ $=V^{\prime}(\phi) / \phi=m^{2}\left[1+K+K \log \left(\phi^{2} / 2 M^{2}\right)\right]$, and still assuming a matter-dominated universe, one finds the same estimates as in the present paper.
}

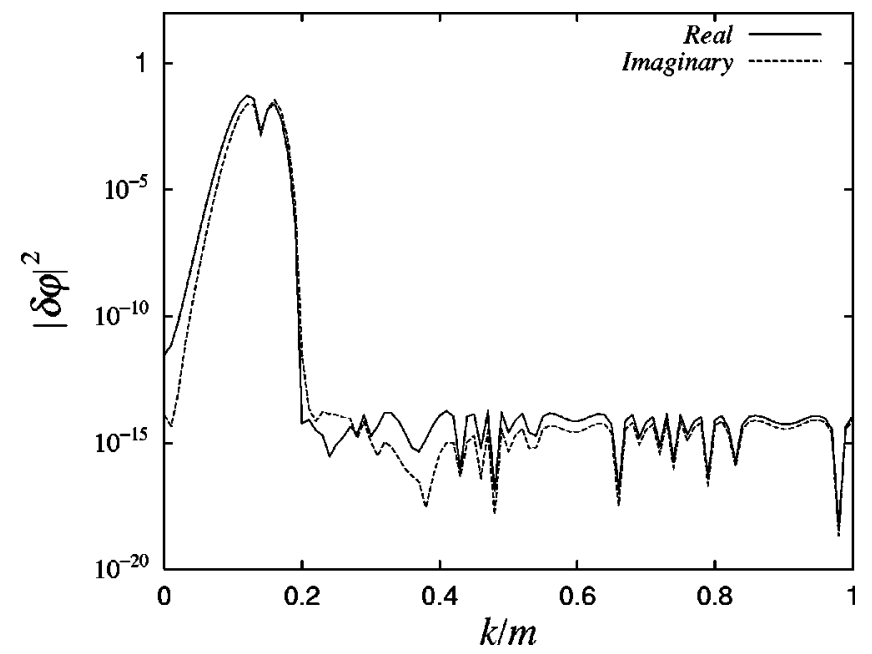

FIG. 2. Instability band when the homogeneous mode is rotating circularly in the potential (1). Solid and dashed lines denote the fluctuations in the real and imaginary directions, respectively. Here we take $K=-0.02$.

$$
V_{12}=V_{21}=2 K \frac{\varphi_{1} \varphi_{2}}{\varphi_{1}^{2}+\varphi_{2}^{2}}
$$

In particular, when the homogeneous part is just oscillating along the radial (i.e., real) direction, we have

$$
\delta \varphi_{i}^{\prime \prime}+3 h \delta \varphi_{i}^{\prime}+\left[\frac{\widetilde{k}^{2}}{a^{2}}+1+\beta_{i} K+K \log \left(\frac{\varphi_{1}^{2}+\varphi_{2}^{2}}{2 M^{2}}\right)\right] \delta \varphi_{i}=0
$$

where $\beta_{1}=3$ and $\beta_{2}=1$. At first glance, we could not see any crucial difference between evolutions of real and imaginary parts of fluctuations. As we see in Fig. 1, however, the imaginary part does not grow at all, while considerable growth can be seen in the real part. (Here we have neglected cosmological expansion for simplicity.) Notice that both real and imaginary parts of the fluctuations develop when the homogeneous part is rotating in the potential. ${ }^{3}$ (See Fig. 2.) In either case, the instability band coincides with the analytical estimate (26).

One might thus be tempted to conclude that there is no $Q$-ball formation, but, as we will see in the next section, mode-mode (self) couplings play a crucial role for developing the imaginary part of the fluctuations, resulting in $Q$ and anti- $Q$ balls to form.

\footnotetext{
${ }^{3}$ When the field is rotating in the potential, fluctuations do not grow for positive $K$. However, the imaginary part of the fluctuations actually grows when the homogeneous field is just oscillating. The growth rate is much larger than that for the negative $K$, but, needless to say, $Q$-ball formation never occurs, since a $Q$-ball solution does not exist for positive $K$.
} 


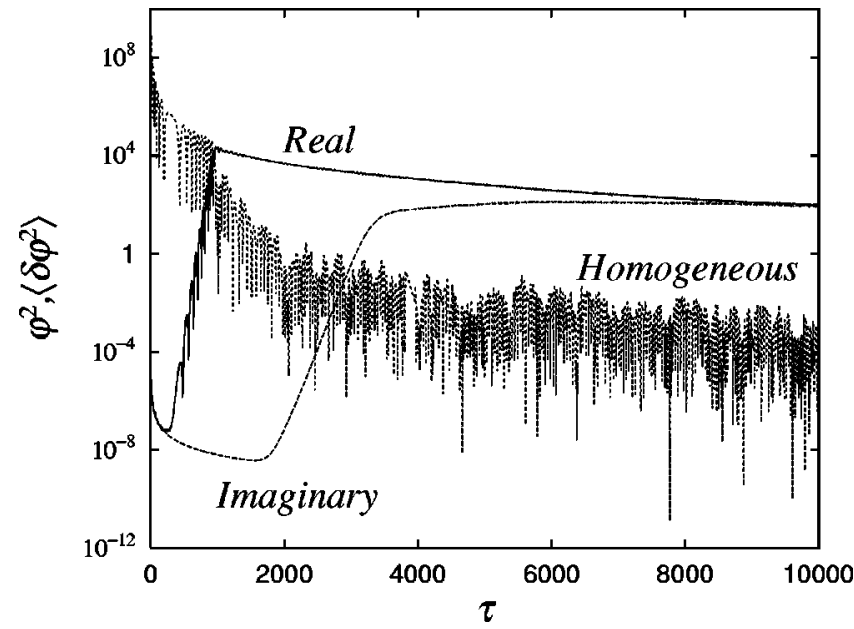

FIG. 3. Evolutions of the amplitudes of homogeneous mode, and fluctuations in the real and imaginary directions.

\section{MODE-MODE COUPLINGS}

It is not apparent that the fluctuations in the imaginary direction grows on the top of the pure oscillating homogeneous condensate in the real direction. As mentioned in the previous section, however, self-couplings of the inflaton field are expected to create fluctuations in the imaginary direction as well. This can be best seen in lattice simulations, which we have done in one, two, and three spatial dimensions. Since, all the cases are more or less the same in the qualitative sense, and the resolution of the three dimensional lattices is not so good, we have grounds for believing that the two-dimensional results presented here will also capture the qualitative behavior of the full three-dimensional case. In the context of Affleck-Dine baryogenesis, lattice simulations have been done previously $[11,16,13,22,23]$, but it is usually assumed that the homogeneous condensate is rotating, not just oscillating in the potential. In addition, here the universe is dominated by the inflaton field in contrast to the AffleckDine case, in which a matter-dominated universe is usually assumed. We also note that $Q$-ball formation in the pure oscillation of the homogeneous condensate has been numerically verified previously in the context of Affleck-Dine mechanism [24], and discussed in the context of quintessence and oscillating inflation [25].

After inflation the inflaton field is almost homogeneous beyond the horizon, and its motion in phase direction has completely frozen. However, there exist small variations, which are the seeds for the large scale structures in the universe. We can thus pose the initial condition at each lattice site as follows:

$$
\begin{aligned}
& \phi_{1}(0)=\phi_{0}+\Delta_{1}, \quad \dot{\phi}_{1}(0)=\dot{\phi}_{0}+\Delta_{2} \\
& \phi_{2}(0)=\Delta_{3}, \quad \dot{\phi}_{2}(0)=\Delta_{4},
\end{aligned}
$$

where $\phi_{0}=M_{P} / \sqrt{12 \pi}, \quad \dot{\phi}_{0}=-m M_{P} / \sqrt{12 \pi}$, and $\Delta$ 's are small numbers which represent small fluctuations. The equations to be integrated are

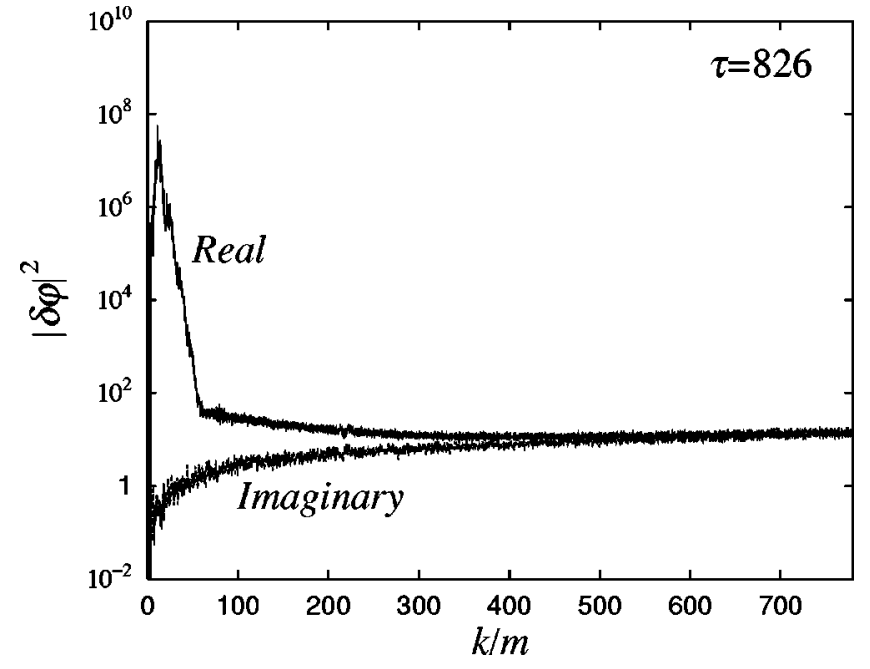

FIG. 4. Power spectra of fluctuations in the real and imaginary directions just before the real part fully develops.

$$
\begin{aligned}
\varphi_{i}^{\prime \prime}+ & 3 h \varphi_{i}^{\prime}-\frac{1}{a^{2}} \nabla^{2} \varphi_{i}+\varphi_{i}[1+K+K \log \\
& \left.\times\left\{\frac{\tilde{m}^{2}\left(\varphi_{1}^{2}+\varphi_{2}^{2}\right)}{2}\right\}\right]=0,
\end{aligned}
$$

where $i=1$ and 2 , which denote real and imaginary parts of the field, respectively, and $\tilde{m}=m / M$. Here we will mainly discuss results from two-dimensional lattices with the box size $N=2048$ and the lattice spacing $\Delta \xi=0.004$, and $K$ $=-0.1$, and show qualitative three-dimensional results with the box size $N=128$, the lattice spacing $\Delta \xi=0.01$, and $K$ $=-0.1$ as well.

Fluctuations in the real direction grow very fast, and their amplitudes become of the same order as that of homogeneous mode at $\tau \sim 1000$. It is depicted in Fig. 3, where we show the time evolution of both real and imaginary parts of the fluctuations. Fluctuations in the imaginary direction, however, have not yet grown at that time, and it starts growing later.

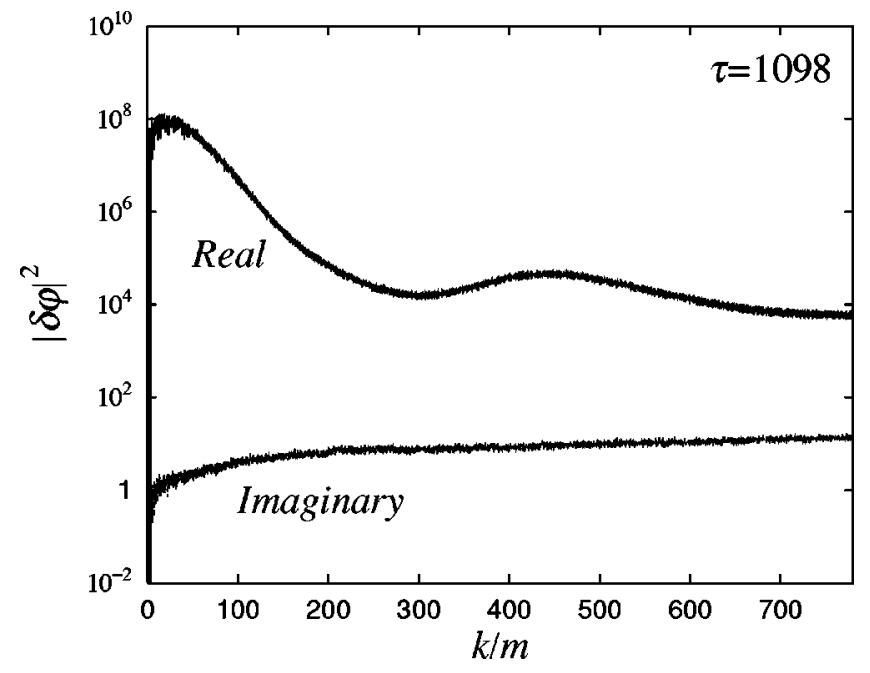

FIG. 5. Power spectra of fluctuations in the real and imaginary directions just after the real part fully developed. 


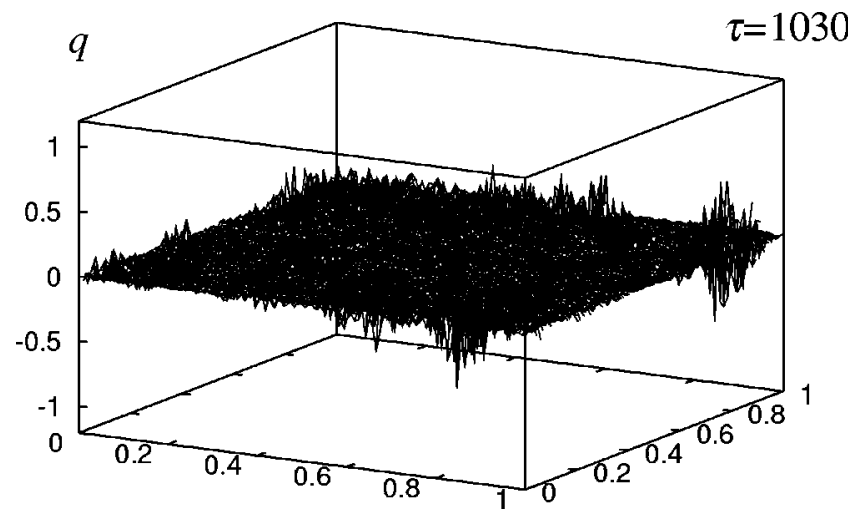

FIG. 6. Charge density of the field. Here we show only a small portion of the entire lattice of $8.192 \times 8.192$.

In Fig. 4, we plot the spectra of real and imaginary parts of the fluctuations at $\tau=826$, just before the former fully develops to the size of the background field value. In fact, the instability band lies in the right range $k \lesssim a(\tau)|K|^{1 / 2} m$, and the most amplified mode coincides with the anticipated $Q$-ball size. After the real part of the fluctuations fully developed, other modes outside the instability band also grow due to mode-mode couplings, as can be seen in Fig. 5, which shows the spectra of the fluctuations at $\tau=1098$, just after the real part has fully developed. Although the spectrum becomes wider and smoother, the position of the most amplified mode, which corresponds the $Q$-ball size, stays still.

One surprising fact is that lumps are actually formed just after the real part of the fluctuations has developed fully even though the imaginary part has not yet started to grow. But, these lumps have not yet contained charges inside. This can be gathered in Figs. 6 and 7, where we respectively show the charge density and the amplitude of the field at the time just after the amplitude of the fluctuations in the real direction catches up with that of the homogeneous mode. Although the field has not settled down completely, we can already see many lumpy objects, which have no charge. Notice that they have in fact the right size of the prospective $Q$ balls: $\sim|K|^{-1 / 2} m^{-1}$.

As time goes on, charges are accumulated into lumps to become $Q$ balls due to mode-mode couplings between fluc-

$$
\tau=1030
$$

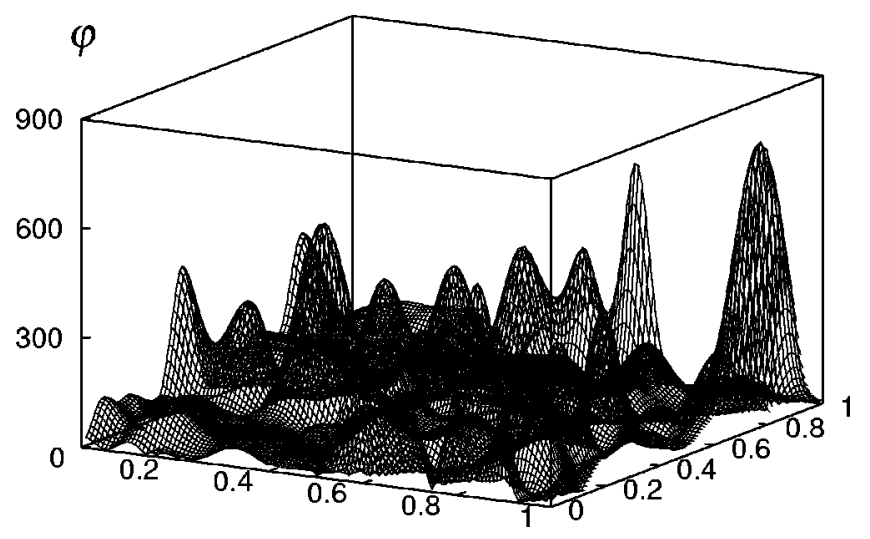

FIG. 7. Amplitude of the field with the same range as in Fig. 6.

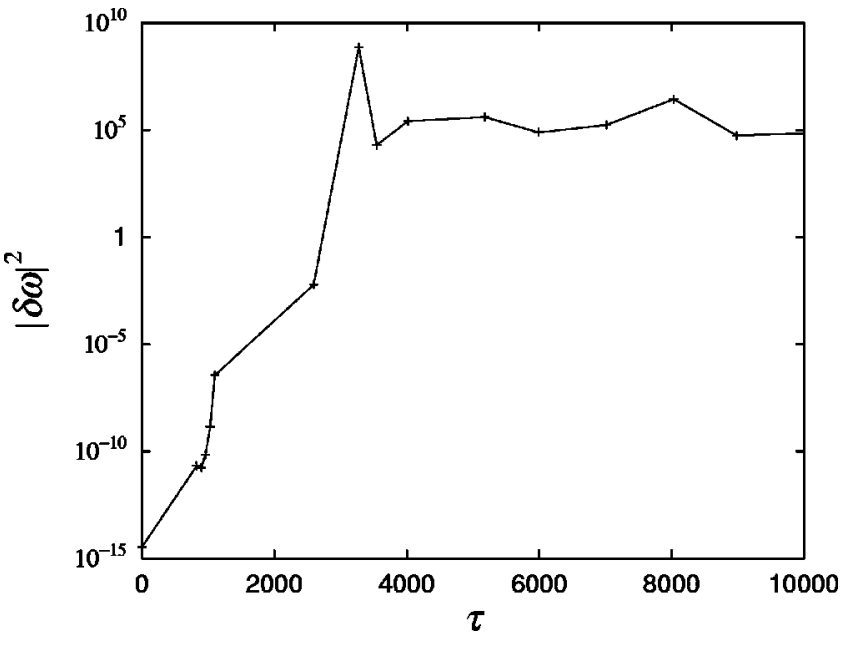

FIG. 8. Evolution of the fluctuation of angular velocity.

tuations in the real and imaginary directions. We show the evolution of $\left\langle\delta \omega^{2}\right\rangle$ in Fig. 8. It is evaluated from

$$
\begin{aligned}
\left\langle\delta \omega^{2}\right\rangle= & \frac{\left[\left(\varphi_{2}^{2}-\varphi_{1}^{2}\right) \varphi_{2}^{\prime}+2 \varphi_{1} \varphi_{2} \varphi_{1}^{\prime}\right]^{2}}{\left(\varphi_{1}^{2}+\varphi_{2}^{2}\right)^{4}}\left\langle\delta \varphi_{1}^{2}\right\rangle \\
& +\frac{\left[\left(\varphi_{2}^{2}-\varphi_{1}^{2}\right) \varphi_{1}^{\prime}-2 \varphi_{1} \varphi_{2} \varphi_{2}^{\prime}\right]^{2}}{\left(\varphi_{1}^{2}+\varphi_{2}^{2}\right)^{4}}\left\langle\delta \varphi_{2}^{2}\right\rangle \\
& +\frac{\varphi_{2}^{2}}{\left(\varphi_{1}^{2}+\varphi_{2}^{2}\right)^{2}}\left\langle\delta \varphi_{1}^{\prime 2}\right\rangle+\frac{\varphi_{1}^{2}}{\left(\varphi_{1}^{2}+\varphi_{2}^{2}\right)^{2}}\left\langle\delta \varphi_{2}^{\prime 2}\right\rangle
\end{aligned}
$$

We see that $\left\langle\delta \omega^{2}\right\rangle$ starts to grow right after the field fluctuations in the real part have fully developed. We may regard it as a local evolution of the charge distribution, or, in other words, the charge accumulation into lumps, which leads to the $Q$-ball formation. Once it is saturated (at $\tau \sim 3500$ ), lumpy objects will not disintegrate but remain as $Q$ balls because of the charge conservation. We can also see in Fig. 9, where the spectra of fluctuations in real and imaginary parts are shown. At around this time, the amplitude of the imaginary part has almost matched with the real partner, which also implies the actual $Q$-ball formation.

At this time, the field values settle down, and $Q$ balls and anti- $Q$ balls are completely isolated from the background. Figures 10 and 11 show the charge density and the amplitude of the field after the imaginary part of the field fluctuation has fully developed. Now we can see "mature" $Q$ balls, and charges of the $Q$ balls conserve from that time on.

Of course, a $Q$-ball formation is seen on the threedimensional lattices. We only show how it appeared in Fig. 12. Here, we plot the configuration at $\tau=3860$, just after $Q$ balls are completely formed. The typical size and charge of the $Q$ balls are $R_{\text {phys }} / m \sim 0.05 \times a(3860) \sim 10$, which almost coincides with the analytically estimated values, and $Q$ $\sim($ a few $) \times 10^{6}$.

Therefore, it appears that in running mass inflation $Q$ balls are produced not only by the negative pressure which makes 


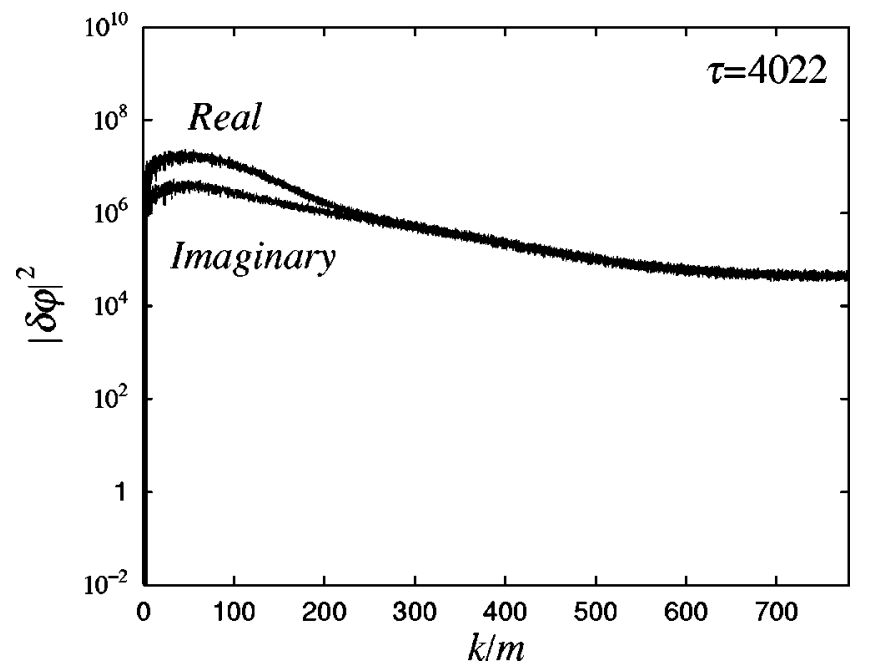

FIG. 9. Power spectra of fluctuations in the real and imaginary directions just after the real part fully developed.

the homogeneous condensate to fluctuate at the same scale as the $Q$-ball size, but the mode-mode coupling plays a crucial role in creating locally distributed charge density. In this sense we can state that in a potential such as Eq. (1) a kind of preheating leads to $Q$-ball formation.

\section{REHEATING THROUGH EVAPORATION}

So far, we have seen it is very natural for the inflaton condensate to deform into $Q$ balls in a certain inflaton potential such as Eq. (1). As a consequence, it may provide the alternative way of reheating after inflation, namely reheating through evaporation, which leads to slower reheating process than usually considered, as we claimed in Ref. [4]. $Q$ balls in the potential (1) are unstable for decaying into particles lighter than $\sim m$ [15]. If the decay products are pairs of fermions, the decay process occurs only on the surface of $Q$ balls because of the Pauli blocking inside the $Q$ ball [18].

A $Q$ ball of size $R \sim|K|^{-1 / 2} m^{-1}$ forms when the fluctuations grow nonlinear. Since the growth rate of fluctuation is $\sim|K| m$, the Hubble parameter at the formation time will be estimated as

$$
H_{f} \sim \gamma|K| m
$$

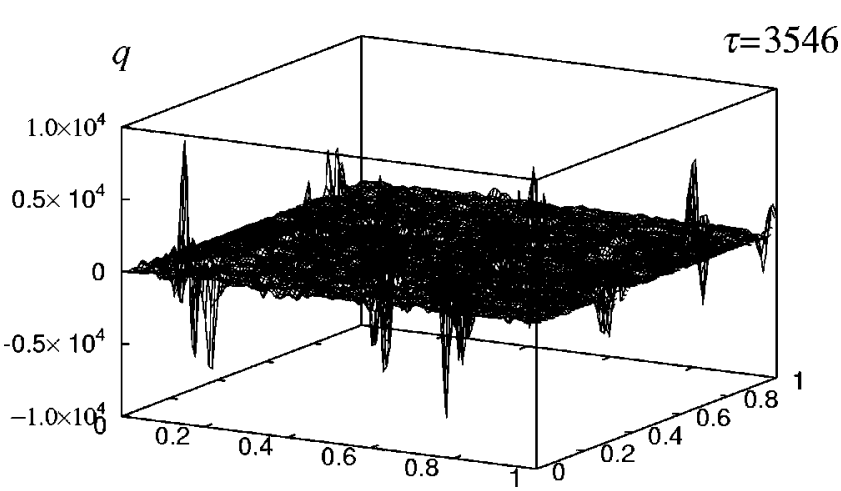

FIG. 10. Charge density distribution in a small sublattice.

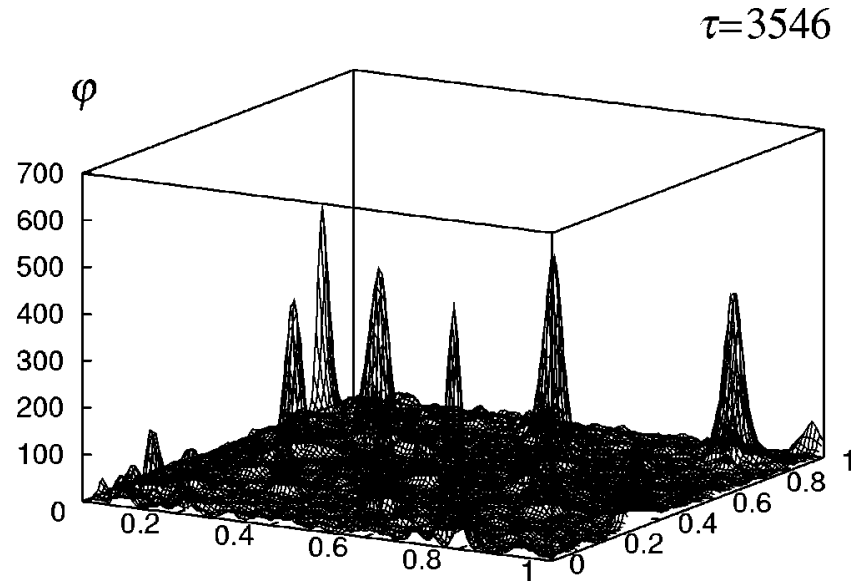

FIG. 11. Amplitude of the field in the same region as in Fig. 10.

where $\gamma \sim 2 \times 10^{-2}$ is some numerical factor derived from numerical simulations. As we assume that $|K| \ll 1$, we can approximate the decrease in the amplitude of the oscillations by $\phi_{f} \sim \phi_{i}\left(H_{f} / H_{i}\right)$ as in the matter dominated era, where $\phi_{i} \simeq M_{\mathrm{P}} / \sqrt{12 \pi}$, denotes the amplitude at the end of inflation in chaotic model, and $H_{i} \sim m$ when the oscillations begin. Therefore, the total charge of a $Q$ ball is given by

$$
Q \sim \frac{4 \pi}{3} R^{3} n_{q} \sim \frac{1}{9} \beta \zeta^{2} \gamma^{2}|K|^{2} R^{3} m M_{\mathrm{P}}^{2}
$$

where $n_{q}=\beta \omega \phi_{0}^{2}, \phi_{0} \simeq \zeta \phi_{f}$, and $\beta \ll 1$ and $\zeta \gtrsim 1$ are numerical factors.

As we shall see below, the evaporating reheat takes over the usual perturbative decay in relatively large coupling where $h \lesssim 1$. In this regime, the evaporation rate is saturated by

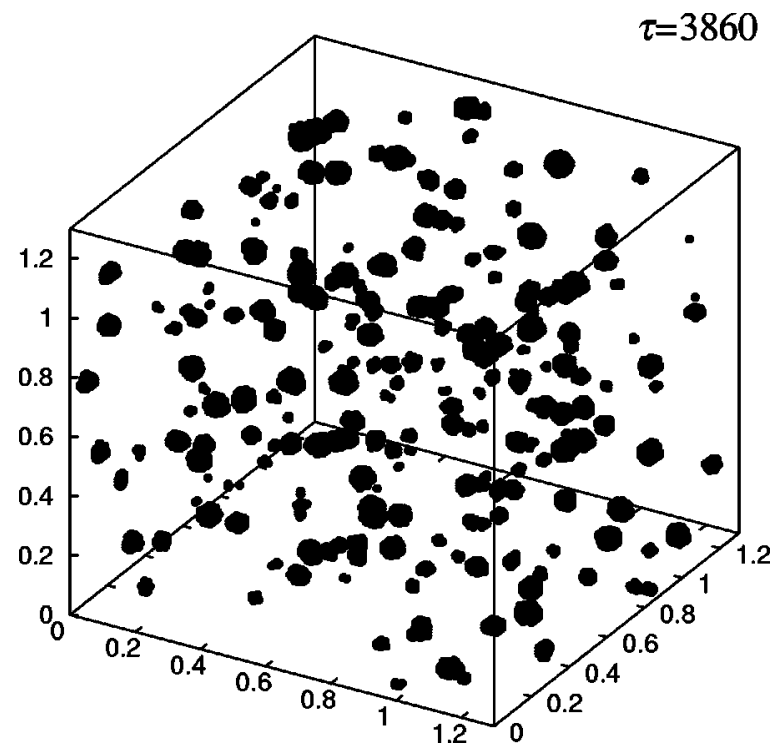

FIG. 12. $Q$ balls in three-dimensional lattices with $N=128$ and $\Delta \xi=0.01$ at $\tau=3860$. 


$$
\frac{d Q}{d t} \lessgtr \frac{\omega^{3} A}{192 \pi^{2}}
$$

where $A \simeq 4 \pi R^{2}$ is the surface area of the $Q$ ball, and $\omega$ $\simeq m$. Thus, we obtain the decay rate of the $Q$ ball through evaporation as

$$
\Gamma_{Q}=\frac{1}{Q} \frac{d Q}{d t} \simeq \frac{3}{16 \pi \beta \zeta^{2} \gamma^{2}|K|^{3 / 2}}\left(\frac{m}{M_{\mathrm{P}}}\right)^{2} m
$$

Note that the decay rate is determined by the ratio $m / M_{\mathrm{P}}$ $\simeq 10^{-6}$, which is fixed by the anisotropies seen in the cosmic microwave background radiation. Therefore, even though we are in relatively large coupling limit, the decay rate mimics that of a Planck suppressed interaction of the inflatonic $Q$ ball to the matter fields.

Now let us compare the rate (37) with the perturbative decay rate $\Gamma_{\text {pert }}=h^{2} m / 8 \pi$. Taking the ratio, we have

$$
\frac{\Gamma_{Q}}{\Gamma_{\text {pert }}} \simeq \frac{3}{2 \beta \zeta^{2} \gamma^{2}|K|^{3 / 2} h^{2}}\left(\frac{m}{M_{P}}\right)^{2} .
$$

When this ratio is less than unity, the evaporation process from the $Q$-ball surface suppresses the reheating. This is achieved if $h \geqslant 10^{-3}$ for reasonable parameters, such as $|K|$ $\sim 0.1, \beta \sim 10^{-2}, \gamma \sim 2 \times 10^{-2}$, and $\zeta \sim 2$. These values of the parameters are derived from numerical simulations on the three-dimensional lattices: $Q \sim 3 \times 10^{6}$, for example. Since the reheating temperature can be estimated as

$$
T_{R H} \simeq 0.1 \sqrt{\Gamma M_{P}} \propto \Gamma^{1 / 2},
$$

it will be suppressed by a factor $\sim 2 \times 10^{-3}$ for $h \sim 1$.

In general $K$ and $h$ are not independent quantities but are related to each other by $|K| \sim C h^{2} / 16 \pi^{2}$, where $C$ is effective number of bosonic and fermionic loops. If the inflaton sector does not belong to a hidden sector, it is very natural that the inflaton coupling to other matter fields is relatively large, i.e. $h \gtrsim\left(m / M_{\mathrm{P}}\right)$. We can thus have easily a situation where $|K|$ $\sim 0.1$, if, e.g., $C \sim 10$ and $h \sim 1$.

To end this section, we will comment on (fermionic) preheating $[26,27]$. In the preheating stage, it is very easy to transport inflaton energy into a pair of massless fermions in an efficient way with the maximum physical momentum

$$
k_{\max } \propto(h \phi m)^{1 / 2},
$$

where $\phi$ is the amplitude of the oscillating inflaton. Even though fermionic preheating is efficient, the whole inflaton energy is not transferred in this process. The energy density stored in the fermions remains small compared to the inflaton energy density, i.e. the comoving number density and the energy density of the massless fermions follow:

$$
n_{f} \sim \frac{k_{\max }^{3}}{6 \pi^{2}} \sim \frac{(h \phi m)^{3 / 2}}{6 \pi^{2}}, \quad \rho_{f} \sim \frac{k_{\max }^{4}}{8 \pi^{2}} \sim \frac{h^{2} \phi^{2} m^{2}}{8 \pi^{2}} .
$$

The ratio of fermion energy density compared to the inflaton energy density after the end of fermion preheating behaves as

$$
\frac{\rho_{f}}{\rho} \sim \frac{h^{2}}{8 \pi^{2}} \ll 1
$$

Hence most of the energy density of the inflaton does not transfer to that of the fermions. This result is quite robust and remains valid even if the fermions have a nonzero bare mass [28].

Even if fermions are produced, they cannot scatter inflaton quanta off its condensate [26], unlike in the case of bosonic preheating with an interaction $g^{2} \phi^{2} \chi^{2}$. The zero mode of the inflaton thus remains intact as a coherent condensate which will fragment into $Q$ balls later. Notice that, as mentioned earlier, a $Q$-ball formation is reminiscent of bosonic preheating due to the presence of attractive self coupling of the inflaton, which stems in the logarithmic term in the potential. Therefore, $Q$-ball formation appears to be a robust feature of any inflationary model with a potential allowing for a $Q$-ball solution. Moreover, for a flatter potential, the size of the $Q$ ball is correlated with the charge, as in the context of the Affleck-Dine condensate with the gaugemediated SUSY breaking. This means that the suppression on the reheating will be much more efficient in those cases.

\section{CONCLUSION AND DISCUSSION}

Inflaton condensate fragmentation and the formation of lumps may be a rather generic, albeit in many cases a transient feature of preheating. However, for the running mass inflaton with $K<0$, and possibly for other types of inflation admitting at least temporarily a mass term growing slower than $|\Phi|^{2}$, the final outcome is markedly different from the usual preheating. The effective equation of state, averaged over field oscillation in the real direction, has negative pressure, which is the cause of the condensate breakup. If the inflaton happens to be a complex field so that it admits soliton solutions carrying a global charge, one would typically expect an inflatonic $Q$ and anti- $Q$ ball pair to be generated when within a $Q$-ball forming region the field fluctuations in both the real and the imaginary directions exceed the coherent background field value.

To verify the generation of inflatonic solitons it is not sufficient to follow only the evolution of the field amplitudes. In the real direction the fluctuations grow very fast to the size of the prospective soliton lumps, but this by itself is not sufficient to ensure $Q$-ball formation. Indeed, field fluctuations in the imaginary direction do not grow at all in the linear approximations. To see what really happens, one has to perform simulations in a configuration space on the lattices, as we have done in the present paper.

In our simulations we can see the formation of lumpy objects but already at relatively early stages when the mean fluctuations in the imaginary direction have not yet developed. These objects have not become $Q$ balls yet, but they eventually accumulate charges to become $Q$ and anti- $Q$ ball pairs. We believe that the reason is that there will be large 
local fluctuations which, once pushed over the limit after which bound solitonic states can exist, cannot fluctuate back. The attractive inflaton self-interaction generates locally distributed $U(1)$ charges through mode-mode couplings which then evolve into semistable inflatonic $Q$ balls. The formation of such solitonic states is nontrivial and stands in contrast to the lumps of the $K>0$ case; the latter is likely to be an evanescent feature that would merely disintegrate and decay away very fast.

Once produced, these $Q$ balls will decay by evaporation and hence give rise to a "slow" reheating because of the suppression which comes from the surface-volume ratio. In particular, this effect becomes more efficient for rather larger coupling $h \sim 1$, which might help the disastrous situations such as the gravitino problem in the supersymmetric scenarios. In fact, the surface evaporation through $Q$ ball is perhaps the most natural way to obtain low reheat temperatures inspite of having order one inflaton coupling to the fermions, which solves not only the gravitino over-abundance problem but also moduli, and dilaton, especially when the inflationary scale is sufficiently large.

Although we have looked upon the running-mass potential, existence of the $Q$-ball solution is a very generic feature in a certain class of models. It would be interesting to study whether such inflatonic $Q$ balls could be seen to arise also in other inflationary models such as hybrid inflation, new inflation, etc., and study in these directions are in progress.

\section{ACKNOWLEDGMENTS}

A.M. is thankful to Rouzbeh Allahverdi, Mar Bastero-Gil and Altug Ozpineci for helpful discussion, A.M. acknowledges the support of The Early Universe network HPRN-CT2000-00152, and the kind hospitality of the Helsinki Institute of Physics where this work has been carried out. K.E. acknowledges the Academy of Finland grant 51433. Part of the numerical calculations was carried out on VPP5000 at the Astronomical Data Analysis Center of the National Astronomical Observatory, Japan.
[1] A.D. Dolgov and A.D. Linde, Phys. Lett. 116B, 329 (1982); L.F. Abbott, E. Farhi, and M.B. Wise, ibid. 117B, 29 (1982).

[2] J.H. Traschen and R.H. Brandenberger, Phys. Rev. D 42, 2491 (1990); L. Kofman, A. Linde, and A.A. Starobinsky, Phys. Rev. Lett. 73, 3195 (1994).

[3] M.Y. Khlopov and A.D. Linde, Phys. Lett. 138B, 265 (1984); J.R. Ellis, J.E. Kim, and D.V. Nanopoulos, ibid. 145B, 181 (1984); M. Kawasaki and T. Moroi, Prog. Theor. Phys. 93, 879 (1995); J. Ellis, D.V. Nanopoulos, K.A. Olive, and S-J. Rey, Astropart. Phys. 4, 371 (1996); for a recent calculation, see M. Boltz, A. Brandenburg, and W. Buchmuller, Nucl. Phys. B606, 518 (2001); for nonperturbative production of gravitino, see A.L. Maroto and A. Mazumdar, Phys. Rev. Lett. 84, 1655 (2000); R. Kallosh, L. Kofman, A. Linde, and A. Van Proeyen, Phys. Rev. D 61, 103503 (2000); R. Allahverdi, M. BasteroGil, and A. Mazumdar, ibid. 64, 023516 (2001); H.P. Nilles, M. Peloso, and L. Sorbo, J. High Energy Phys. 04, 004 (2001).

[4] K. Enqvist, S. Kasuya, and A. Mazumdar, hep-ph/0204270.

[5] S. Coleman, Nucl. Phys. B262, 263 (1985); T.D. Lee and Y. Pang, Phys. Rep. 221, 251 (1982).

[6] G. Felder, J. Garcia-Bellido, P.B. Greene, L. Kofman, A. Linde, and I. Tkachev, Phys. Rev. Lett. 87, 011601 (2001); G. Felder, L. Kofman, and A. Linde, Phys. Rev. D 64, 123517 (2001); E.J. Copeland, S. Pascoli, and A. Rajantie, ibid. 65, 103517 (2002).

[7] J. McDonald, hep-ph/0105235.

[8] I.A. Affleck and M. Dine, Nucl. Phys. B249, 361 (1985).
[9] G. Dvali, A. Kusenko, and M. Shaposhnikov, Phys. Lett. B 417, 99 (1998).

[10] A. Kusenko and M. Shaposhnikov, Phys. Lett. B 418, 46 (1998).

[11] S. Kasuya and M. Kawasaki, Phys. Rev. D 61, 041301 (2000).

[12] S. Kasuya and M. Kawasaki, Phys. Rev. Lett. 85, 2677 (2000).

[13] S. Kasuya and M. Kawasaki, Phys. Rev. D 64, 123515 (2001).

[14] K. Enqvist and J. McDonald, Phys. Lett. B 418, 46 (1998).

[15] K. Enqvist and J. McDonald, Nucl. Phys. B538, 321 (1999).

[16] S. Kasuya and M. Kawasaki, Phys. Rev. D 62, 023512 (2000).

[17] D. Lyth and A. Riotto, Phys. Rep. 314, 1 (1999).

[18] A. Cohen, S. Coleman, H. Georgi, and A. Manohar, Nucl. Phys. B272, 301 (1986).

[19] M.S. Turner, Phys. Rev. D 28, 1243 (1983).

[20] J. McDonald, Phys. Rev. D 48, 2573 (1993).

[21] A. Jokinen, hep-ph/0204086.

[22] T. Multamäki and I. Vilja, Phys. Lett. B 535, 170 (2002).

[23] K. Enqvist, A. Jokinen, T. Multamäki, and I. Vilja, Phys. Rev. D 63, 083501 (2001).

[24] S. Kasuya, in Proceedings of the 7th International Symposium on Particles, Strings and Cosmology (PASCOS-99), Lake Tahoe, 1999, edited by K. Cheung et al. (World Scientific, Singapore, 2000), p. 301.

[25] S. Kasuya, Phys. Lett. B 515, 121 (2000).

[26] J. Baacke, K. Heitmann, and C. Patzold, Phys. Rev. D 58, 125013 (1998).

[27] P. Greene and L. Kofman, Phys. Lett. B 448, 6 (1999).

[28] G.F. Giudice, M. Peloso, A. Riotto, and I. Tkachev, J. High Energy Phys. 08, 014 (1999). 